\title{
UNDERSTANDING THE GEOMETRY OF ASTROPHYSICAL MAGNETIC FIELDS
}

\author{
AVERY E. BRODERICK ${ }^{1} \&$ ROger D. BlandFORD ${ }^{2}$ \\ ${ }^{1}$ Canadian Institute for Theoretical Astrophysics, 60 St. George St., Toronto, ON M5S 3H8, Canada; aeb@ cita.utoronto.ca \\ ${ }^{2}$ Kavli Institute for Particle Astrophysics and Cosmology, 2575 Sand Hill Rd., Menlo Park, CA 94309, USA \\ Draft version October 23, 2018
}

\begin{abstract}
Faraday rotation measurements have provided an invaluable technique with which to measure the properties of astrophysical magnetized plasmas. Unfortunately, typical observations provide information only about the density-weighted average of the magnetic field component parallel to the line of sight. As a result, the magnetic field geometry along the line of sight, and in many cases even the location of the rotating material, is poorly constrained. Frequently, interpretations of Faraday rotation observations are dependent upon underlying models of the magnetic field being probed (e.g., uniform, turbulent, equipartition). However, we show that at sufficiently low frequencies, specifically below roughly $13\left(\mathrm{RM} / 1 \mathrm{radm}^{-2}\right)^{1 / 4}(B / 1 \mathrm{G})^{1 / 2} \mathrm{MHz}$, the character of Faraday rotation changes, entering what we term the "super-adiabatic regime" in which the rotation measure is proportional to the integrated absolute value of the line-of-sight component of the field. As a consequence, comparing rotation measures at high frequencies with those in this new regime provides direct information about the geometry of the magnetic field along the line of sight. Furthermore, the frequency defining the transition to this new regime, $\nu_{\mathrm{SA}}$, depends directly upon the local electron density and magnetic field strength where the magnetic field is perpendicular to the line of sight, allowing the unambiguous distinction between Faraday rotation within and in front of the emission region. Typical values of $\nu_{\mathrm{SA}}$ range from $10 \mathrm{kHz}$ (below the ionospheric cutoff, but above the heliospheric cutoff) to $10 \mathrm{GHz}$, depending upon the details of the Faraday rotating environment. In particular, for resolved AGN, including the black holes at the center of the Milky Way (Sgr A*) and M81, $\nu_{\mathrm{SA}}$ ranges from roughly $10 \mathrm{MHz}$ to $10 \mathrm{GHz}$, and thus can be probed via existing and up-coming ground-based radio observatories. Subject headings: polarization - radiative transfer - radio continuum: general - magnetic fields - turbulence Galaxy: center
\end{abstract}

\section{INTRODUCTION}

Magnetized plasmas are common in astrophysics, playing central roles in objects as diverse as galaxy clusters, the sites of star formation, the solar corona and accreting black holes and their ultra-relativistic outflows. Despite their importance, however, there are few observational tools for assessing the physical conditions within them. This is especially true for strongly ionized or diffuse regions, in which molecular line emission is absent. In these the best evidence for significant magnetic fields, beyond theoretical arguments, is due to Faraday rotation observations of intrinsically polarized sources.

First discovered in optically active crystals (Faraday 1846), Faraday rotation has subsequently become one of the primary methods for measuring the strength and geometry of astrophysical magnetic fields. This has been primarily via the determination of the rotation measure, defined in terms of the polarization angle, $\Phi$, by

$$
\mathrm{RM}=\frac{d \Phi}{d \lambda^{2}} \simeq 8.12 \times 10^{5} \int n_{e} \mathbf{B} \cdot d \ell \mathrm{radm}^{-2},
$$

where $n_{e}, B$ and $\ell$ are measured in $\mathrm{cm}^{-3}, \mathrm{G}$ and pc, respectively. Thus, RM's provides a line-of-sight averaged measurement of the line-of-sight magnetic field, weighted by the local plasma density. Measured values for the RM range from nearly zero to nearly $10^{6} \mathrm{rad} \mathrm{m}^{-2}$.

Rotation measure observations have played a critical role in determining the magnetic field strengths in the centers of galaxy clusters (e.g., Ge \& Owen 1993, 1994), Galactic magnetic field strength and distribution (e.g., Men et al. 2008; Noutsos et al. 2008), magnetic field strength and structure within ultra-relativistic black hole jets (Zavala \& Taylor 2004; Stirling et al. 2004; Miller-Jones et al. 2005; Brocksopp et al.
2007; Kharb et al. 2009), magnetic field strengths near, and accretion rate of, the supermassive black hole at the center of the Milky Way and other nearby low-luminosity active galactic nuclei (AGN) (e.g., Agol 2000; Quataert \& Gruzinov 2000; Brunthaler et al. 2006; Macquart et al. 2006; Marrone et al. 2007), and even the solar corona magnetic field (e.g., Mancuso \& Spangler 2000).

However, there are a number of significant ambiguities in Faraday rotation studies. The first is the degeneracy between density and magnetic field strength (i.e., high densities and weak fields vs. low densities and strong fields). The second is the degeneracy between weak large-scale fields and strong tangled fields. In light of numerical simulations of magnetohydrodynamic turbulence, and the observation of supersonic (though sub-Alfvénic) turbulence in Galactic molecular clouds, these make interpreting measured RM's highly dependent upon models for the rotating medium. Since in many cases it is unclear if the Faraday rotation is occuring in situ or in a foreground region distant from the polarized source, there are large uncertainties in the our understanding of the physical conditions in the source.

Here we show that at low frequencies Faraday rotation qualitatively changes, with the RM becoming proportional to $\int n_{e}|\mathbf{B} \cdot d \ell|$. Comparisons between RM's measured within this "super-adiabatic" regime and at high frequencies can probe the line-of-sight geometry of the magnetic field ${ }^{1}$. Furthermore, the frequency at which a given source transitions between the standard and super-adiabatic regimes depends upon the local plasma properties at magnetic field reversals (when the mag-

\footnotetext{
1 Analogous phenomena include the "MSW" effect in neutrino astrophysics (Bahcall 1989)
} 
netic field is orthogonal to the line of sight), and can thus distinguish between different candidates for the site of the rotating medium.

In Section 2 we review the plasma processes responsible for Faraday rotation and describe the super-adiabatic regime. Section 3 deals with the observational consequences unique to super-adiabatic Faraday rotation generally, while Sections 4 \& 5 concern specific classes of sources in which ordinary Faraday rotation has been observed. Our conclusions and implications are summarized in Section 6. The mathematical details of an $a b$ initio determination of the radiative transfer regimes is presented in the Appendix.

\section{FARADAY ROTATION \& ADIABATIC PLASMA MODE PROPAGATION}

Faraday rotation is commonly presented in terms of the different phase velocities of the circularly polarized eigenmodes of magnetized plasmas. Specifically, a linearly polarized, monochromatic, incident wave is decomposed into the two plasma modes, which subsequently propagate independently, accruing a net phase difference, $\Delta \Phi=\int \Delta k d \ell$ (where $\Delta k(\omega)$ is the difference between the wave-vectors of the two modes), as a consequence of the anisotropic nature of magnetized plasmas. This phase difference between circularly polarized modes results in a net rotation of the polarization angle by $\Delta \Phi / 2$. Frequently, this is extended to the case of elliptical plasma modes, which occurs when $\mathbf{B}$ is nearly orthogonal to the line of sight, or if the electrons are relativistic, producing Faraday conversion (also know as Faraday pulsations or generalized Faraday rotation), corresponding to oscillations between linear and circular polarizations as well as a rotation of the polarization angle.

Implicit in this description of the plasma processes underlying Faraday rotation is the assumption that the two plasma eigenmodes do indeed propagate independently, or "adiabatically", i.e., there is no mode crossing. In the case of uniform plasmas (constant $n_{e}, \mathbf{B}$ ) this is true, and the foregoing explanation is complete. However, if the plasma is rapidly changing the situation is more complicated. For example, consider the propagation of the fast mode near the location of a magnetic field reversal, i.e., where $\mathbf{k} \cdot \mathbf{B}$ changes sign. Initially, the mode is nearly circularly polarized, with the electric vector rotating in the same sense as the gyrating electrons. As the component of the magnetic field decreases the mode becomes increasingly elliptical, changing into the linearly-polarized extraordinary mode when $\mathbf{k} \cdot \mathbf{B}=0$. On the other side of the magnetic reversal the mode again becomes elliptical and eventually circular, though now with the sense of rotation reversed, following the sense of gyration of the electrons. Similarly, the slow mode reverses its polarization by evolving through the linearly polarized ordinary mode. The net Faraday rotation is then dependent upon $\int n_{e}|\mathbf{B} \cdot d \boldsymbol{\ell}|$. Clearly the conditions for the applicability of Equation (1) depend upon adiabaticity being broken at field reversals. We call this the "standard" regime, with the regions where the adiabatic condition fails referred to as the "strongly coupled". However, the "super-adiabatic" regime, where the propagation is adiabatic throughout field reversals, can sometimes be observed with interesting consequences.

Since the failure of the adiabatic condition occurs near field reversals, we might expect that the standard and superadiabatic regimes are distinguished by the properties of the eigenmodes when $\mathbf{B} \cdot \mathbf{k}=0$. Specifically, we might expect that when the eigenmodes change sufficiently slowly at field

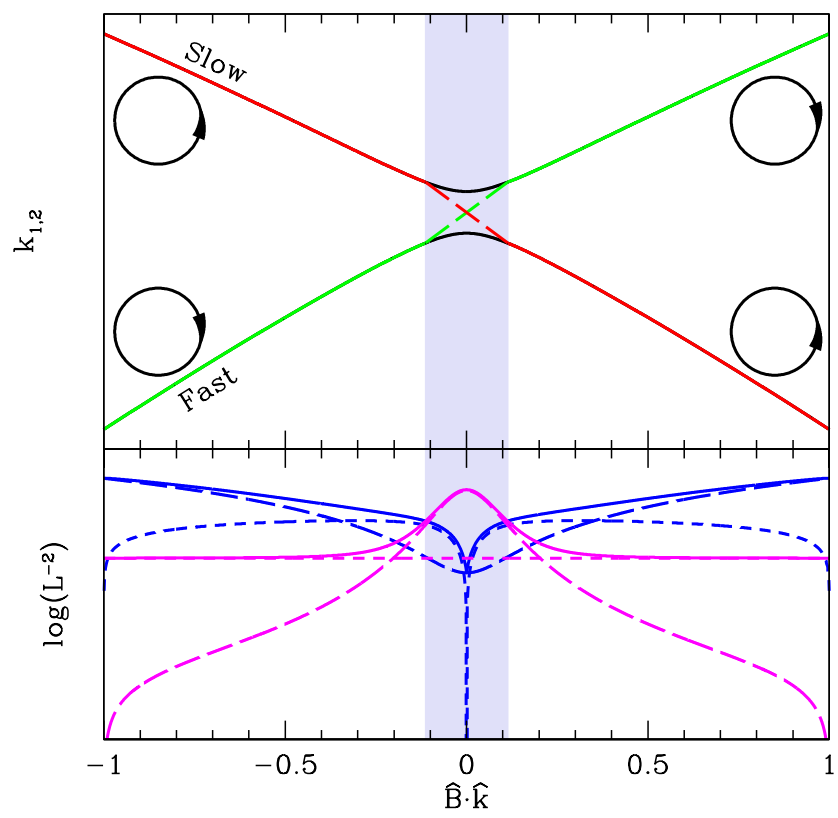

FIG. 1.- Mode behavior near magnetic field reversals. Top: The wave numbers of the two plasma eigenmodes, with their quasi-longitudinal polarizations shown. Within the strongly coupled regime the amplitudes of the two modes effectively switch, producing the standard expression for the rotation measure: $\mathrm{RM}_{\mathrm{S}} \propto \int n_{e} \mathbf{B} \cdot d \boldsymbol{\ell}$. Bottom: The terms in the adiabatic condition (Equation 2). Blue long dash, short dash and solid lines show $(\Delta k / 2)^{2},\left|\Delta k^{\prime} / 2\right|$ and their sum, respectively. Magenta long dash, short dash and solid lines show $\chi^{\prime 2}, \phi^{\prime 2}$ and their sum, respectively. When the latter is larger than the former the propagation occurs in the strongly coupled regime, denoted by the shaded region, and significant mode conversion can occur.

reversals, the propagation remains adiabatic. Near reversals the evolution of the eigenmodes are dominated by the increase in ellipticity, becoming significantly elliptical only when $|\theta-\pi / 2| \lesssim Y \equiv \nu_{B} / \nu$, where $\theta$ is the angle between the magnetic field and the line of sight and $\nu_{B}$ is the electron cyclotron frequency. Thus, the eigenmodes change substantially on length scales on the order of $Y(d \theta / d \ell)^{-1}$ near reversals. To ignore mode conversion we require that this be large in comparison to $\Delta k^{-1}$, which at reversals is given approximately by $c / \pi \nu X Y^{2}$, where $X \equiv \nu_{P}^{2} / \nu^{2}$ in which $\nu_{P}$ is the electron plasma frequency ${ }^{2}$. Thus we arrive heuristically at the result that when $c d \theta / d \ell \ll \pi \nu X Y^{3}$ the propagation occurs in the superadiabatic regime.

More explicitly, as shown in the Appendix, the necessary condition for the local plasma modes to propagate adiabatically is

$$
\left(\frac{\Delta k}{2}\right)^{2}+\left|\frac{\Delta k^{\prime}}{2}\right| \gg \phi^{\prime 2}+\chi^{\prime 2},
$$

where prime denotes differentiation with respect to $\ell, \phi$ and $\chi$ are the orientation and ellipticity angle of the polarization ellipse, respectively. However, to second order in the plasma parameters $\Delta k$ is given by

$$
\Delta k=2 \pi \frac{\nu}{c} X Y \sqrt{\cos ^{2} \theta+\left(\frac{Y}{2}\right)^{2} \sin ^{2} \theta}
$$

\footnotetext{
2 Equivalently, we require the energy of the mixing term associated with the evolution of the underlying eigenmodes be less than the energy splitting between the two modes
} 


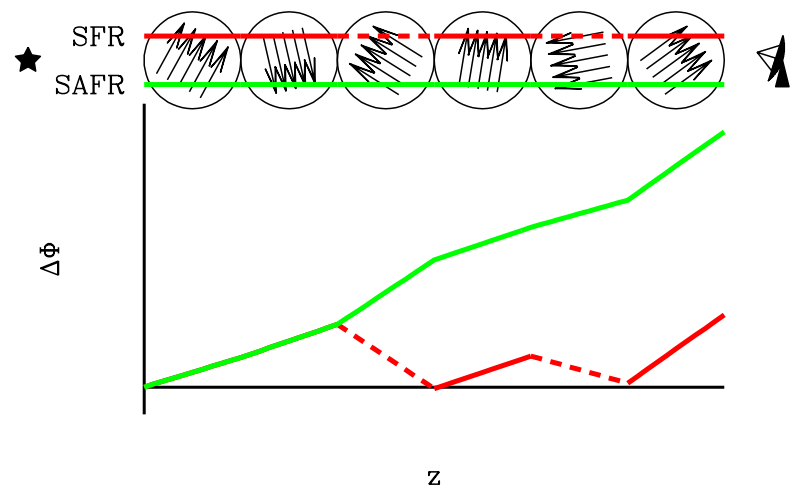

FIG. 2.- The growth the phase difference between the two plasma eigenmodes during propagation through a turbulent medium. Intervening cells of roughly uniform magnetic field with different orientations and strengths are shown on the top, with the source on the left and the observer on the right. The red line shows a path taken during standard Faraday rotation, with the phase difference accruing during solid segments and decrementing during dashed segments. The green line shows a similar path taken during super-adiabatic Faraday rotation, with the phase difference always accruing. Note the considerable enhancement of $\Delta \Phi$ in the super-adiabatic regime, resulting in a corresponding enhancement in the rotation measure in this regime.

where $\theta$ is the angle between the magnetic field and the line of sight. When $\mathbf{B} \perp \mathbf{k}, \Delta k$ is reduced by a factor of $Y / 2$ from its quasi-longitudinal value, and $\Delta k^{\prime}$ vanishes. On the other hand, near $\theta=\pi / 2$, the eigenmode ellipticity changes rapidly, giving:

$$
\chi^{\prime} \simeq \frac{Y}{4} \frac{\sin \theta\left(1+\cos ^{2} \theta\right)}{(Y / 2)^{2}+\cos ^{2} \theta} \theta^{\prime},
$$

where we have assumed $Y \ll 1$. If we further assume that the direction of rotation of the magnetic field is roughly isotropic, $\theta^{\prime} \simeq \phi^{\prime}$, implying that at reversals $\chi^{\prime} \simeq \phi^{\prime} / Y$. Thus at the same time, the left side of Equation (2) falls substantially, the right side increases by a similar factor. This is illustrated explicitly in the bottom panel of Figure 1, which shows the left-side (blue) and right-side (magenta) of the adiabatic condition through a reversal. As a consequence, at magnetic field reversals the mode propagation almost always becomes strongly coupled, and in the limit of small $Y$ the polarization propagates as in vacuum.

A direct result of the failure of the adiabatic condition at magnetic field reversals is that the left-handed (for concreteness) fast mode maps onto the left-handed slow mode on the other side of the reversal, and vice versus. That is, there is an almost complete conversion of one mode to the other. Thus, we have the situation shown by the red line in Figure 2, as the polarized wave passes through regions of magnetic field with $\mathbf{B} \cdot \mathbf{k}>0, \Delta \Phi$ increases (solid lines), while when $\mathbf{B} \cdot \mathbf{k}<0, \Delta \Phi$ decreases (dashed lines), yielding the well-known formula,

$$
\Delta \Phi \propto \nu^{-2} \int n_{e} \mathbf{B} \cdot d \boldsymbol{\ell}
$$

Note that the failure of the adiabatic condition at field reversals was critical to obtaining the standard result.

Nevertheless, at sufficiently low frequencies, the adiabatic condition, Equation (2), can be satisfied at reversals. That is, because $\left|\Delta k^{\prime}\right|$ vanishes and $\chi^{\prime} \gg \phi^{\prime}$ where $\mathbf{B} \cdot \mathbf{k}=0$, in order to remain in the adiabatic mode propagation regime throughout, what we call the "super adiabatic" regime, we require

$$
\frac{\theta^{\prime}}{Y} \ll \frac{\pi}{2} \frac{\nu}{c} X Y^{2} \Rightarrow \nu \ll \nu_{\mathrm{SA}} \equiv\left[\frac{\pi \nu_{B}^{3} \nu_{P}^{2}}{2 c \theta^{\prime}}\right]^{1 / 4}
$$

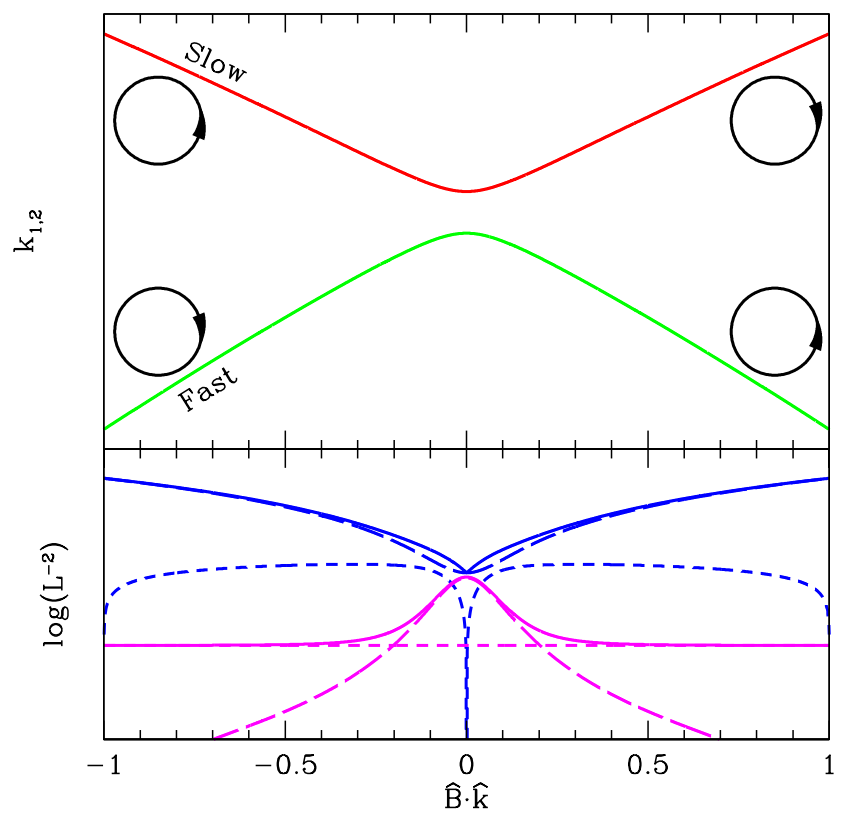

FIG. 3.- Mode behavior near magnetic field reversals in the super-adiabatic regime. Top: Same as in Figure 1, except now the modes are no longer coupled near $\mathbf{B} \cdot \mathbf{k}=0$. As a result, the fast/slow mode remains the fast/slow mode, though the local polarizations reverse, and $\mathrm{RM}_{\mathrm{SA}} \propto \int n_{e}|\mathbf{B} \cdot d \boldsymbol{\ell}|$. Bottom: Same as in Figure 1, though note the absence of a strongly coupled regime.

Note that up to a factor of two, this is precisely the condition we reached earlier by heuristic arguments. The critical frequency below which the propagation is super-adiabatic may be recast in terms of the local magnetic field reversal length scale, $\ell_{B} \equiv$ $\pi / 2 \theta^{\prime} \equiv 10^{15} \ell_{B, 15} \mathrm{~cm}$, and the local plasma parameters:

$$
\nu_{\mathrm{SA}} \simeq 87 n^{1 / 4} B^{3 / 4} \ell_{B, 15}^{1 / 4} \mathrm{MHz},
$$

in which $n$ and $B$ are measured in $\mathrm{cm}^{-3}$ and G, respectively. Alternatively, this may be written in terms of the total number of reversals along the line of sight, $N \simeq \theta^{\prime} L / 2 \pi$, the rotation measure determined at frequencies above $\nu_{\mathrm{SA}}, \mathrm{RM}_{\mathrm{S}} \equiv \lambda^{-2} \Delta \Phi \simeq$ $\left.\lambda^{-2} \Delta k\right|_{\theta=0} L / \sqrt{N}$ (where $N$ enters due to the cancellations discussed previously) and the magnetic field strength:

$$
\begin{aligned}
\nu_{\mathrm{SA}} & \simeq\left(\frac{\nu_{B} c}{2 \sqrt{2 \pi}}\right)^{1 / 2} \mathrm{RM}_{\mathrm{S}}{ }^{1 / 4} N^{-1 / 8} \\
& \simeq 13 B^{1 / 2} \mathrm{RM}_{\mathrm{S}}{ }^{1 / 4} N^{-1 / 8} \mathrm{MHz}
\end{aligned}
$$

where in the final expression $B$ and $\mathrm{RM}_{\mathrm{S}}$ are in $\mathrm{G}$ and $\mathrm{radm}^{-2}$, respectively. Note that this is only a weak function of $\mathrm{RM}_{S}$ and $N$, and thus primarily indicative of the local magnetic field strength in the source. As we shall see in Section 4, for a number of sources this produces $\nu_{\mathrm{SA}}$ near $100 \mathrm{MHz}-1 \mathrm{GHz}$.

Since in the super-adiabatic regime there is no mode crossing at magnetic field reversals, the fast/slow mode maps onto the fast/slow mode, as illustrated in Figure 3. As a result, the phase difference between the modes only accrues, as seen by the green line in Figure 2. That is, for super-adiabatic Faraday rotation,

$$
\Delta \Phi_{\mathrm{SA}} \propto \nu^{-2} \int n_{e}|\mathbf{B} \cdot d \boldsymbol{\ell}|
$$




\section{OBSERVATIONAL CONSEQUENCES OF SUPER-ADIABATIC FARADAY ROTATION}

There are three additional fundamental constraints upon observable values of $\nu_{\mathrm{SA}}$. The first is the requirement that within the Faraday screen, $\nu_{\mathrm{SA}}$ must exceed the upper cutoff of the extraordinary mode:

$$
\nu_{\mathrm{SA}}>\frac{\nu_{B}}{2}\left(1+\sqrt{1+4 \frac{\nu_{P}^{2}}{\nu_{B}^{2}}}\right) .
$$

Inserting the Equation (8) and assuming that there is considerable separation between the cyclotron and plasma scale gives two conditions upon the magnetic field,

$$
B>2.1 \times 10^{-5} \mathrm{RM}_{\mathrm{S}}{ }^{1 / 4} N^{-1 / 8} \ell_{B, 15}^{1 / 2} \mathrm{G},
$$

corresponding to $\nu_{\mathrm{SA}}>\nu_{P}$, and

$$
B<22 \mathrm{RM}_{\mathrm{S}}{ }^{1 / 2} N^{-1 / 4} \mathrm{G},
$$

corresponding to $\nu_{\mathrm{SA}}>\nu_{B}{ }^{3}$. Note that this produces lower and upper and bound upon $B$ as a function of $\mathrm{RM}_{\mathrm{S}}$. The former is shown for various values of $N^{-1 / 4} \ell_{B}$ by the dashed lines in Figure 4. The latter is denoted by the upper-left greyed region in Figure 4.

The second is that we must be able to ignore difference in the refraction of the two plasma eigenmodes. As the plasma eigenmodes propagate through the Faraday screen they necessarily follow different paths, dictated by their different indices of refraction. If the two paths pass through sufficiently distinct regions of the Faraday screen, Equation (1) is no longer valid, $\Phi$ depending upon the differences in the plasma conditions along the paths in addition to the difference in the phase velocities of the two modes, and the RM is no longer constant. As shown in the Appendix, the condition that the modes propagate through strongly correlated plasma regions is $\nu \gg \nu_{\mathrm{R}}$ where

$$
\begin{aligned}
\nu_{\mathrm{R}} & \simeq 61 \frac{D^{1 / 3} L^{1 / 6}}{\ell_{B}^{1 / 2}} n^{1 / 3} B^{1 / 3} f^{1 / 3} \mathrm{kHz} \\
& \simeq 2.0 D_{22}^{1 / 3} \ell_{B, 15}^{-2 / 3} \mathrm{RM}^{1 / 3} f^{1 / 3} \mathrm{MHz}
\end{aligned}
$$

in which $f$ is the fractional variation in the plasma parameters, $D=\left(D_{O}^{-1}+D_{S}^{-1}\right)^{-1}=D_{22} 10^{22} \mathrm{~cm}$ where $D_{O}$ and $D_{S}$ are the observer-screen and source-screen distances, respectively. The nature of this constraint depends upon the location of the Faraday screen. For example, when the rotation occurs in situ, $D \simeq L$ implying that $D / \ell_{B} \simeq N$, and giving $\nu_{R}$ that is a moderately weak function of $N\left(\propto N^{1 / 2}\right.$ or $N^{1 / 3}$ depending upon which of the above expressions one considers). For all of the sources we describe below, $\nu_{\mathrm{SA}}>\nu_{\mathrm{R}}$, justifying our neglect of refraction for these objects.

The third is the observational limits due to absorption in the ionosphere and heliopause (see, e.g., Bougeret 1996). The ionosphere limits ground observations to above roughly $15 \mathrm{MHz}$, though down to $10 \mathrm{MHz}$ is possible at excellent sites. A fundamental limit for space missions is the plasma frequency at the heliopause, estimated at roughly $9 \mathrm{kHz}$. These are shown by the hatched and solid grey regions, respectively, in the lower left. While a number of interesting objects lie within reach of ground-based radio astronomy, including nearby AGN and pulsars, pushing to space-based, ultra-low frequency radio observatories opens a new window upon a vast array of astrophysical environments.

\footnotetext{
${ }^{3}$ This also implies that we may ignore Razin suppression.
}

If it can be observed, Faraday rotation in the super-adiabatic regime, what we term "super-adiabatic Faraday rotation", provides a wealth of new information about the magnitude and line-of-sight geometry of magnetic fields. First, unlike standard Faraday rotation the super-adiabatic rotation measure:

$$
\mathrm{RM}_{\mathrm{SA}}=C \int n_{e}|\mathbf{B} \cdot d \boldsymbol{\ell}|,
$$

does not suffer from a degeneracy between weak large-scale magnetic fields and strong tangled magnetic fields. Thus, $\mathrm{RM}_{\mathrm{SA}}$ is far better suited for the determination of $n_{e}$ and $B$ via equipartition arguments than its high-frequency analog:

$$
\mathrm{RM}_{\mathrm{S}}=C \int n_{e} \mathbf{B} \cdot d \boldsymbol{\ell} .
$$

Second, generally, $\mathrm{RM}_{\mathrm{SA}}$ will be greater than $\mathrm{RM}_{\mathrm{S}}$ by an amount depending upon the number of magnetic field reversals along the line of sight. Typically, we may expect that looking through $N$ independent turbulent cells, the excess phase difference, and therefore $\mathrm{RM}_{\mathrm{S}}$, to scale as $N^{-1 / 2}$, i.e.,

$$
\mathrm{RM}_{\mathrm{SA}} \simeq N^{1 / 2} \mathrm{RM}_{\mathrm{S}} .
$$

Thus, measuring both $\mathrm{RM}_{\mathrm{S}}$ (at $\nu>\nu_{\mathrm{SA}}$ ) and $\mathrm{RM}_{\mathrm{SA}}\left(\right.$ at $\nu<\nu_{\mathrm{SA}}$ ) provides an estimate for the number of magnetic field reversals along the line of sight:

$$
N \simeq\left(\frac{\mathrm{RM}_{\mathrm{SA}}}{\mathrm{RM}_{\mathrm{S}}}\right)^{2}
$$

However, this estimate may not be appropriate in the presence of long-range order in the magnetic field, and more than a rough estimate requires a detailed analysis of the nature of the magnetic fields under consideration. For example, if there was some reason to not expect a randomly reversing field, but instead a band-structured field (e.g., as might be expected from pulsars or hydromagnetic turbulence within a given cell), then, $\mathrm{RM}_{\mathrm{SA}} / \mathrm{RM}_{\mathrm{S}} \simeq N$.

Third, as seen in Equation (8), the location of the superadiabatic transition depends upon the local parameters of the plasma, though we have recast them in terms of the known $\mathrm{RM}_{\mathrm{S}}$ and $B$ by assuming that the intervening Faraday screen isn't significantly clumpy. However, in many sources this assumption may not be appropriate. Measuring $\nu_{\text {SA }}$ provides a direct measurement of the local plasma properties at the magnetic field reversals, and thus the distribution of the electron density and magnetic field strength along the line of sight. On the other hand, if we can assume that these quantities are smoothly distributed, measuring $\mathrm{RM}_{\mathrm{SA}}$ and $\nu_{\mathrm{SA}}$ provides a method to estimate the magnetic field strength, independent of the intervening electron density:

$$
B \simeq 6 \nu_{\mathrm{SA}}^{2}\left(\frac{\mathrm{RM}_{\mathrm{SA}}}{\mathrm{RM}_{\mathrm{S}}}\right)^{1 / 4} \mathrm{RM}_{\mathrm{S}}{ }^{-1 / 2} \mathrm{mG},
$$

where $\nu_{\mathrm{SA}}$ and $\mathrm{RM}_{\mathrm{S}}$ are measured in $\mathrm{MHz}$ and $\mathrm{radm}^{-2}$, respectively.

However, both bandwidth and beam depolarization are likely to be more severe problems for super-adiabatic Faraday rotation than its standard counterpart. Both are exacerbated simply because $\mathrm{RM}_{\mathrm{SA}}>\mathrm{RM}_{\mathrm{S}}$. Beam depolarization, however, is also more likely due to the nature of super-adiabatic propagation. At field reversals the polarization is effectively reflected about the magnetic field as the handedness of the polarization eigenmode switches. The observed polarization is 
therefore dependent in part upon the magnetic field direction in the last turbulent cell. This can produce large gradients in the polarization position angle on angular scales comparable to the turbulent scale. Furthermore, because probing the superadiabatic regime requires pushing to longer wavelengths, it also generically results in larger beams. Nevertheless, for compact sources, those that subtend a small angular scale in comparison to the turbulence in the intervening Faraday screen, this effect is largely mitigated.

\section{IMPLICATIONS FOR SPECIFIC SOURCES: GROUND BASED OBSERVATIONS}

We now turn to the implications for specific classes of sources, and in particular estimate $\nu_{\mathrm{SA}}$. Figure 4 summarizes these. Here we dicuss those sources for which the superadiabatic regime is accessible from ground based observations, i.e., $\nu_{\mathrm{SA}} \gtrsim 15 \mathrm{MHz}$.

\subsection{Ionospheric Faraday Rotation}

The ionosphere presents an opportunity to investigate the super-adiabatic regime via local radio experiments. The Flayer is roughly $300 \mathrm{~km}$ high with a typical free-electron density of $10^{6} \mathrm{~cm}^{-3}$. Using a typical terrestrial magnetic field strength of $0.5 \mathrm{mG}$, this results in an RM of roughly $4 \mathrm{rad} \mathrm{m}^{-2}$ at zenith, and potentially considerably more at oblique angles. This will be further enhanced at low frequencies by ionospheric refraction. Thus, while moderate in comparison to most astronomical sources, the atmospheric rotation measure is certainly detectable.

Near the poles the Earth's magnetic field necessarily changes direction, with $\theta^{\prime} \simeq \pi / R_{\oplus}$. Thus, $\nu_{\mathrm{SA}} \simeq 40 \mathrm{MHz}$, which is well above the ionospheric cut-off. While ionospheric refraction is significant at this frequency, the differential refraction is sufficiently small $\left(\nu_{R} \simeq 5 \mathrm{MHz}\right)$. Thus, transpolar observations of the Jovian or Saturnian decametric radiation provides a natural terrestrial radio experiment in which to study the transition from the standard to the super-adiabatic regimes in a well understood plasma.

\subsection{Resolved Galactic Nuclei}

\subsubsection{Sagittarius $A^{*}$}

The supermassive black hole at the center of the Milky Way, Sagittarius $A^{*}\left(\operatorname{Sgr} A^{*}\right)$, is presently the best studied accreting black hole. Despite its comparatively small mass $\left(4.7 \times 10^{6} M_{\odot}\right.$, Ghez et al. 2008; Gillessen et al. 2009) and extraordinarily low luminosity $\left(L / L_{\mathrm{Edd}} \simeq 10^{-10}\right)$, due to its proximity it has the unique distinction of being the only black hole to have been probed on sub-horizon scales (Doeleman et al. 2008). It also has the largest rotation measure observed, $-5.6 \pm 0.7 \times 10^{5} \mathrm{rad} \mathrm{m}^{-2}$ (Marrone et al. 2007; Macquart et al. 2006). Estimates of Sgr A*'s magnetic field arise from both fitting simple accretion models to both the rotation measure results and the source spectrum (Agol 2000; Quataert \& Gruzinov 2000; Falcke \& Markoff 2000; Yuan et al. 2003; Marrone et al. 2007; Loeb \& Waxman 2007), and range from 0.01$100 \mathrm{G}$. The considerable uncertainty reflects the uncertainty in the location region responsible for the Faraday rotation as well as the geometry of the magnetic field along the line of sight. Nevertheless, these estimates imply that Sgr A* should enter the super-adiabatic regime between $30 \mathrm{MHz}$ and $3 \mathrm{GHz}$.

Typical length scales within the Faraday rotating medium surrounding Sgr A* are on the order of $\ell_{B} \simeq 10^{3} \mathrm{GM} / \mathrm{c}^{2} \simeq$
$10^{15} \mathrm{~cm}$. Even with the large RM's observed in Sgr A*, this still results in a $\nu_{\mathrm{R}} \simeq 1 \mathrm{MHz}$, and thus refractive decoherence is unimportant.

$$
\text { 4.2.2. } M 81 *
$$

The supermassive black hole at the center of M81 is a second representative of a class of low-luminosity AGN. At $7 \times 10^{6} M_{\odot}$, it is moderately more massive that Sgr A*, and with $L / L_{\text {Edd }} \simeq 10^{-5}$, considerably brighter, though still considerably sub-Eddington (Brunthaler et al. 2006). The lack of polarization in M81 at wavelengths above $3.6 \mathrm{~mm}$ implies lower limits upon the value of RM of $10^{4} \mathrm{rad} \mathrm{m}^{-2}$ and $4 \times 10^{5} \mathrm{rad} \mathrm{m}^{-2}$, depending upon whether the source is beam or band depolarized (Brunthaler et al. 2006). Estimates for the magnetic field in the Faraday screen are again made by appealing to modeling the spectrum of M81* and in analogy with Sgr A* (see above), giving a range of roughly $2-2 \times 10^{4} \mathrm{G}$, due to its larger luminosity and mass. This corresponds to somewhat larger $\nu_{\mathrm{SA}}$, near $3 \mathrm{GHz}$, though closer to the $\nu_{\mathrm{SA}} \simeq \nu_{B}$ cutoff. Again refraction is unimportant in this source.

\subsubsection{Other Resolved AGN}

Determining the magnetic field strengths within the Faraday screen of nearby AGN other than Sgr A* and M81* is the primary difficulty in assessing the likelihood of observing super-adiabatic Faraday rotation in these sources. For other resolved, sub-Eddington AGN we may estimate the magnitude of the magnetic field in the accretion flow via scaling arguments, equipartition and Sgr A*. Specifically, in terms of the luminosity in Eddington units, $\ell_{\mathrm{Edd}}=L / L_{\mathrm{Edd}} \propto \dot{M} / \dot{M}_{\mathrm{Edd}}$, the electron (and therefore ion) density near the black hole scales as

$$
n_{e} \propto \frac{\dot{M}}{M^{2} c} \propto \ell_{\mathrm{Edd}} M^{-2} \dot{M}_{E d d} \propto \ell_{\mathrm{Edd}} M^{-1},
$$

where we have assumed that the radiative efficiency is not a strong function of mass for substantially sub-Eddington sources. The ion temperature is largely independent of black hole mass, reaching $10^{11} \mathrm{~K}$ in nearly all cases. Therefore, the equipartition magnetic field strength scales as

$$
B \propto\left(n_{e} k T\right)^{1 / 2} \propto \ell_{\mathrm{Edd}}^{1 / 2} M^{-1 / 2} .
$$

We can also use this to estimate the expected scaling of rotation measures:

$$
\mathrm{RM} \propto n_{e} B d \ell \propto \ell_{\mathrm{Edd}}^{3 / 2} M^{-1 / 2} .
$$

Note that these imply that brighter and lower-mass sources will have higher values of $\nu_{\mathrm{SA}}$. Since the rotation occurs locally in these sources, $\nu_{\mathrm{SA}} / \nu_{\mathrm{R}} \propto \ell_{\mathrm{Edd}}^{1 / 2} M^{1 / 4}$, and thus refractive decoherence is unlikely to ever preclude observing the super-adiabatic regime for resolved AGN.

The black hole at the center of M31 is shown as an example. Despite being much more massive, $M \simeq 1.4 \times 10^{8} M_{\odot}$, it also is extraordinarily under-luminous, with $\ell_{\text {Edd }} \simeq 10^{-10}$, similar to Sgr A* (Li et al. 2009). The correspondingly rescaled RM and $B$ is shown in Figure 4. More generally, polarization observations of resolved nuclear regions will fill the upper-right corner of the $B-\mathrm{RM}$ plane, corresponding to typical $\nu_{\mathrm{SA}}$ ranging from the ground-based cutoff of $15 \mathrm{MHz}$ to $10 \mathrm{GHz}$.

\subsection{Unresolved AGN}

Faraday rotation has been one of the primary methods for studying the magnetic geometry of AGN radio jets and cores. 


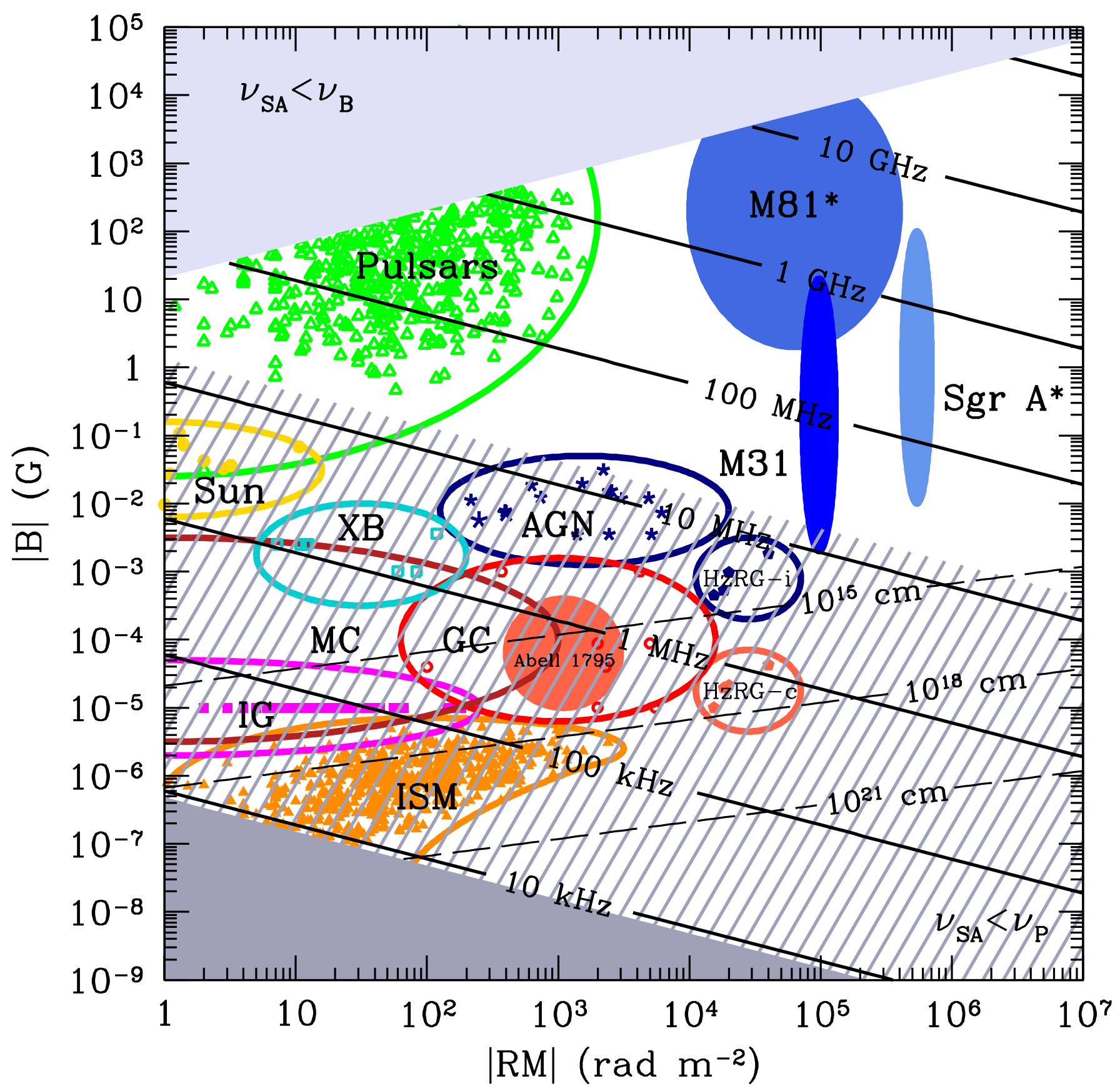

FIG. 4. - Lines of constant $\nu_{\mathrm{SA}}$ for various rotation measures and source magnetic field strengths, assuming $N=1$ (though since $\nu_{\mathrm{SA}}$ is so weakly dependent upon $N$ it is unlikely to be significantly different in practice). We require that $\nu_{\mathrm{SA}}$ lie above the upper-cutoff frequency, which reduces to roughly the condition that $\nu_{\mathrm{SA}}>\nu_{B}$ and $\nu_{\mathrm{SA}}>\nu_{P}$ (see Equations $11 \& 12$ ). The first of these corresponds to $B<22 \mathrm{RM}_{\mathrm{S}}{ }^{1 / 2} N^{-1 / 4} \mathrm{G}$, and is violated within the upper-left grayed region. The second reduces to $B>21 \mathrm{RM}_{S}{ }^{1 / 4} N^{-1 / 8} \ell_{B}^{1 / 2} \mu \mathrm{G}$ and is shown by the thin dashed lines in the lower-right for $\ell_{B}=10^{15} \mathrm{~cm}, 10^{18} \mathrm{~cm}$ and $10^{21} \mathrm{~cm}$. The lower-left grey-hatched region denotes where $\nu_{\mathrm{SA}}$ lies below the ionospheric cutoff $(\sim 15 \mathrm{GHz})$ and the low-left greyed region denotes where $\nu_{\mathrm{SA}}$ lies below the heliospheric cutoff $(\sim 10 \mathrm{kHz})$. The locations, and therefore the expected $\nu_{\mathrm{SA}}$, for various classes of potential sources are shown, based upon observed RM's and estimated magnetic field strengths. Points and filled ellipses correspond to individual sources, while the empty regions are rough estimates of the envelope bounding the region each class of sources occupies. The represented source classes, and the sections discussing them in more detail, are: Sgr A* (4.2.1), M81 (4.2.2), M31 (4.2.3), AGN cores \& jets and high-redshift radio galaxies (AGN \& HzRG-i, 4.3), intrinsic rotation in Pulsars (Pulsars, 4.4), the solar corona (Sun, 5.1), the interstellar medium (ISM, 5.2), X-ray binaries (XB, 5.3), Galactic center region (GC, 5.4), Galactic molecular clouds (MC, 5.5), infrared/starburst galaxies (IG, 5.6) and the intracluster medium (Abell 1795 \& HzRG-c, 5.7). 
RM gradients have even produced direct evidence for a helical field geometry in AGN jets (see, e.g. Kharb et al. 2009). Presently, rotation measures exist for more than 40 AGN, ranging from roughly $10^{2} \mathrm{rad} \mathrm{m}^{-2}$ to nearly $10^{4} \mathrm{rad} \mathrm{m}^{-2}$ (Zavala \& Taylor 2004). These are usually determined via fitting VLBA observations at $8 \mathrm{GHz}$ and $15 \mathrm{GHz}$, and in nearly all cases does not resolve the central source. In principle, these are associated with the accreting black hole or jet, and thus are in many respects similar to the systems discussed in Section 4.2, though generally with considerably higher $\ell_{\text {Edd }}$.

Estimating the magnetic field is again the primary challenge. We obtain an approximate magnetic field strength based upon an equipartition argument with the emitting nonthermal electrons, assumed to be accelerated in relativistic shocks either in the accretion flow or in the jet. In this case we expect the electron power-law index to be roughly -2 between some minimum and maximum Lorentz factors, and $L_{\nu}$ is given locally by

$$
L_{\nu} \simeq 3 \times 10^{32} \gamma_{\min } n_{e} B^{3 / 2} V \nu^{-1 / 2} \mathrm{erg} \mathrm{s}^{-1} \mathrm{~Hz}^{-1},
$$

where $n_{e}, B, V$ and $\nu$ are given in $\mathrm{cm}^{-3}, \mathrm{G}, \mathrm{pc}^{3}$ and $\mathrm{GHz}$, respectively (the flat spectrum of AGN are then produced by spatial structure in these quantities, see, e.g., Blandford \& Konigl 1979). The electron pressure is given by

$$
P_{e} \simeq \frac{1}{3} \epsilon_{e}=\frac{1}{3} \gamma_{\min } m_{e} c^{2} n_{e} \ln \left(\frac{\gamma_{\max }}{\gamma_{\min }}\right)
$$

If the ion pressure is similar, equipartition gives $P_{e} \simeq \beta B^{2} / 8 \pi$, for typical $\beta$ 's of 10-100. Thus, upon combining Equations (22) \& (23), given $\nu, L_{\nu}$, and $V$, we obtain a lower limit upon the field strength:

$$
B \simeq 2 \times 10^{2}(\Lambda / \beta)^{-2 / 7} L_{\nu, 35}^{2 / 7} \nu^{1 / 7} V^{-2 / 7} \mathrm{mG},
$$

where $L_{\nu}=10^{35} L_{\nu, 35} \mathrm{erg} \mathrm{s}^{-1} \mathrm{~Hz}^{-1}, \Lambda \equiv \ln \left(\gamma_{\max } / \gamma_{\min }\right)$ and the other quantities are given in the units described previously. Note that this is weakly dependent upon $\beta$, and thus the system would need to be extraordinarily sub-equipartition for the magnetic field to be appreciably lower. For concreteness we choose $\Lambda \simeq 10$ and $\beta \simeq 10^{2}$.

For the AGN jets \& cores for which we found RM's in the literature (Zavala \& Taylor 2004; Kharb et al. 2009), at $\nu=15 \mathrm{GHz}, L_{\nu, 35}$ is typically of order unity. More difficult is estimating $V$, since the vast majority of AGN cores are unresolved, and we can therefore only place an upper limit upon $V$ (lower limit upon the equipartition value of $B$ ). To estimate this, for each AGN we inspected high-frequency VLBI images to determine approximate upper limits upon its angular size, typically comparable to beam sizes of $\sim 1$ mas (Taylor 1998; Lister et al. 1998; Taylor 2000; Lister \& Smith 2000; Lister 2001; Porcas \& Rioja 2002; Zavala \& Taylor 2003, 2004; Jorstad et al. 2005; Lister et al. 2009). Given the distances inferred from the source redshifts, this corresponds to limits upon the physical sizes of roughly $5 \mathrm{pc}$, or volumes of $10^{2} \mathrm{pc}^{3}-$ $10^{3} \mathrm{pc}^{3}$. The resulting estimates for the magnetic field strength are $3 \mathrm{mG}-30 \mathrm{mG}$, and shown by the dark-blue region labeled "AGN" in Figure 4.

In addition to the AGN discussed above, there are a small number of high-redshift radio galaxies ( $\mathrm{H} z \mathrm{RG})$ exhibiting anomalously large rest-frame rotation measures. These include OQ $172\left(z=3.535, \mathrm{RM} \simeq 4 \times 10^{4} \mathrm{rad} \mathrm{m}^{-2}\right.$, Udomprasert et al. 1997), 3C $295\left(z=3.377, \mathrm{RM} \simeq 2 \times 10^{4} \mathrm{rad} \mathrm{m}^{-2}\right.$, Perley \& Taylor 1991; Taylor \& Perley 1992), SDSS 1624+3758 $\left(z=0.461, \mathrm{RM} \simeq 1,8 \times 10^{4} \mathrm{rad} \mathrm{m}^{-2}\right.$, Frey et al. 2008), and PKS B0529-549 $\left(z=2.575, \mathrm{RM} \simeq 1.5 \times 10^{4} \mathrm{rad} \mathrm{m}^{-2}\right.$, Broderick et al.
2007). These RM are an order of magnitude larger than those discussed previously, and thus may represent a distinct class. Their association with cooling-core clusters has led to speculation that the Faraday rotation is associated with the intracluster medium and not intrinsic to the broad-line region. Nevertheless, if the rotation does occur within the core, this implies typical magnetic fields of $0.5 \mathrm{mG}$, somewhat lower than those for other AGN due to their comparatively larger distances. This is shown in the dark-blue region labeled "HzRG-i".

Resolved AGN exhibit strong RM gradients, frequently with RM dispersions that exceed the averaged RM. As a result, the RM for unresolved AGN cores is likely to be considerably smaller than the peak values. Furthermore, as we have stressed above, our estimates of the local magnetic field are highly uncertain. This is primarily a consequence of the unresolved natures of most AGN, implying that our estimate of the equipartition magnetic field is only a lower limit. If the Faraday rotation is occuring near the supermassive black hole, as appears to be the case in $\mathrm{Sgr} \mathrm{A}^{*}$, then the AGN region should move up and to the right. Therefore, while for AGN typical $\nu_{\mathrm{SA}}$ 's presently lie just at the frequency cutoff for groundbased observations, in reality it may be closer to those for their resolved counterparts. Furthermore, for in situ rotation, $\nu_{\mathrm{R}} \simeq 0.3 N^{1 / 2} \ell_{B, 18}^{-1 / 3} \mathrm{RM}_{3}^{1 / 3} \mathrm{MHz}$, still comfortably below $\nu_{\mathrm{SA}}$.

On the other hand, if the Faraday screen is far from the region responsible for the emission, the intrinsic magnetic field may be closer to $1 \mu \mathrm{G}-100 \mu \mathrm{G}$, typical of the interstellar medium. In this case, the $\nu_{\mathrm{SA}}$ 's would lie well below the ground-based cutoff, though above the heliospheric cutoff, implying that in these sources super-adiabatic Faraday rotation may be observable from ultra-low frequency, space-based radio observatories. Thus, even just the detection of super-adiabatic Faraday rotation would determine the region responsible for the observed Faraday rotation.

\subsection{Pulsars: Intrinsic Rotation}

The rotation measures of pulsars can be quite large, reaching $3 \times 10^{3} \mathrm{rad} \mathrm{m}^{-2}$ in some sources. Traditionally, this has been interpreted to be due to Faraday rotation in the interstellar medium (ISM, see Section 5.2). Addressing the propagation of radio waves through pulsar magnetospheres has been studied extensively elsewhere (see, e.g., Arons \& Barnard 1986; Barnard \& Arons 1986; Thompson et al. 1994), and is beyond the scope of this paper. Generally, it has been found that the propagation modes are quite different than those we have described here, due to structure within the magnetosphere and the presence of a dominant population of relativistic particles. Nevertheless, it is not clear that propagation through the plerion does not result in a substantial contribution to the observed RM's.

Within the plerion, the wind is again dominated by relativistic particles, which have strongly elliptical polarization modes, complicating the determination of the expected RM's. In addition, the wind has a relativistic bulk motion, which can significantly alter Equation (1), (see, e.g., Broderick \& Loeb 2009). For objects like the Crab, for which the bulk Lorentz factor can reach $10^{6}$, these relativistic effects can dominate the Faraday rotation, depending upon where the Faraday rotating medium is located.

Given these caveats, observing super-adiabatic Faraday rotation in pulsars would present direct evidence for intrinsic Faraday rotation in pulsars, with the attendant consequences for the geometry of pulsar magnetospheres and recalibrating Galac- 
tic magnetic field studies. Given the uncertainties mentioned above, it is unclear what the relevant magnetic field strength is in this case. Here we take as a fiducial value that at the light cylinder, $B_{\mathrm{lc}} \simeq B_{\text {surf }}\left(R_{\mathrm{NS}} / R_{\mathrm{lc}}\right)^{3}=\left(2 \pi R_{\mathrm{NS}} / P c\right)^{3}$, typically on the order of $10 \mathrm{G}-10^{3} \mathrm{G}$, though in some cases considerably higher. While the magnetic field strength is a strong function of radius (though a considerably weaker function in the wind region, where $B$ is roughly proportional to $r^{-1}$, than in the magnetosphere in which $B \propto r^{-3}$ ), it does not reach interstellar values until roughly $3 \times 10^{-6} \mathrm{pc}$, vastly larger than the light cylinder. If the entire measured RM is due to the region near the pulsar, the implied magnetic field strengths would place pulsars in the upper-left of Figure 4 for 749 objects, collected from Noutsos et al. (2008) and the ANTF catalog at www.atnf.csiro.au/research/pulsar/psrcat (Manchester et al. 2005), shown in green and labeled "Pulsars".

\section{IMPLICATIONS FOR SPECIFIC SOURCES: SPACE BASED OBSERVATIONS}

Despite lying below the ionospheric cutoff, the superadiabatic regime for many objects is potentially accessible from space (e.g., Jones et al. 2000). These necessarily have $\nu_{\mathrm{SA}} \gtrsim 9 \mathrm{kHz}$, and are thus above the heliospheric cutoff. However, in many cases they are considerably closer to their respective refractive decoherence limits. Nevertheless, the observation of the super-adiabatic regime provides a prime motivation to push towards a space-based, low-frequency radio emission.

\subsection{Solar Corona}

Faraday rotation measurements of polarized radio sources near the solar disk provide a unique method with which to probe the magnetic field of the solar corona. Within this region the solar magnetic field is still quite strong, ranging from $10 \mathrm{mG}$ to $0.2 \mathrm{G}$, and dense, with an electron density on the order of $10^{3} \mathrm{~cm}^{-3}$. Nevertheless, the short path length results in only modest RM's, ranging between $10 \mathrm{radm}^{-2}$ and zero (Mancuso \& Spangler 2000). These imply that $\nu_{\mathrm{SA}} \simeq 3 \mathrm{MHz}$, shown by the yellow region labeled "Sun" in Figure 4 . The refractive limit is roughly $0.4 D_{11}^{1 / 3} \ell_{B, 11}^{-2 / 3} \mathrm{RM}_{1}^{1 / 3} \mathrm{MHz}$, and thus roughly an order of magnitude below this. Therefore, potential low-frequency, space-based radio observatories could directly probe the Corona for large-amplitude, long-wavelength Alfvén waves, a leading contender for the mechanism responsible for heating the solar corona. Alternatively, observing the refractive decoherence, characterized by a sustained departure from the $\lambda^{2}$-law, can place a direct limit upon the scale of the dominant wave modes.

\subsection{Pulsars: Interstellar Medium}

Traditionally, the observed RM of pulsars has been attributed to the propagation through the ISM. When coupled with a determination of the dispersion measure, Pulsar RM's provide a probe of the strength and distribution of the Galactic magnetic field (see, e.g., Men et al. 2008; Noutsos et al. 2008). Typical inferred magnetic field strengths for the ISM are on the order of $1 \mu \mathrm{G}-10 \mu \mathrm{G}$, and place Pulsar polarization measurements in the orange region labeled "ISM" in the lower-left of Figure 4 for 749 objects, collected from Noutsos et al. (2008) and the ANTF catalog at www.atnf.csiro.au/research/pulsar/psrcat (Manchester et al. 2005). For many pulsars, this extends above the heliospheric cutoff, reaching in some cases $100 \mathrm{kHz}$. However, less well defined is the refractive decoherence limit, which depends critically upon the turbulent scale of the ISM. That is, $\nu_{\mathrm{R}} \simeq 10 D_{22}^{1 / 3} \ell_{B, 20}^{-2 / 3} \mathrm{RM}_{3}^{1 / 3} \mathrm{kHz}$, and thus as long as the ISM varies primarily on scales above $30 \mathrm{pc}$ refraction may be ignored. On the other hand, sub-parsec turbulence in the ISM could push $\nu_{\mathrm{R}}$ above $200 \mathrm{kHz}$, rendering the super-adiabatic regime inaccessible.

\subsection{X-ray Binaries}

As the stellar mass analogs of AGN, X-ray binaries might be expected to produce sizable RM's. This is supported by our RM estimate for accreting black holes. With typical luminosities and masses on the order of $10^{-1} L_{\text {Edd }}$ and $10 M_{\odot}$, respectively, Equation (21) implies tremendous rotation measures, $\mathrm{RM} \simeq 8 \times 10^{21} \mathrm{rad} \mathrm{m}^{-2}$. Therefore, Faraday rotation within the accretion flow will almost certainly be band-depolarized below the $\mathrm{X}$-rays (i.e., for $\lambda \lesssim 3 \mathrm{~A}$ given a resolving power of 500). Despite this, the transition into the super-adiabatic regime doesn't occur until roughly $40 \mathrm{eV}$, making it all but impossible to probe the accretion flow in this way. However, the magnetic fields and densities in X-ray binary jets are considerably lower, and there have been a handful of radio RM measurements of these features.

At $\mathrm{GHz}$ frequencies, SS 433's jets are polarized at the $1 \%$ level, and have a rotation measure of roughly $-119 \mathrm{radm}^{-2}$ (Gilmore \& Seaquist 1980; Stirling et al. 2004). The magnetic field strength in the region surrounding the jets, inferred from fitting the source structure at $5 \mathrm{GHz}$, is roughly $4 \mathrm{mG}$, considerably weaker than that associated with the core $(>20 \mathrm{mG})$ though much stronger than the ISM field.

During outbursts, GRS 1915+105 and XTE J1748-288 have both exhibited substantial polarizations, rising in both cases above $20 \%$ level. In GRS $1915+105$, during these periods, the polarization angle of the ejected component evolved in a fashion consistent with rotation measures of $60 \mathrm{radm}^{-2}-80 \mathrm{radm}^{-2}$ (Miller-Jones et al. 2005). While it isn't clear what the strength of the magnetic field is in this source, estimates based upon equipartition arguments in the ejected jet give $B \simeq 1 \mathrm{mG}$. For XTE J1748-288, on the other hand, the rotation measure varied between $7 \mathrm{rad} \mathrm{m}^{-2}-12 \mathrm{rad} \mathrm{m}^{-2}$, over a 10 day period (Brocksopp et al. 2007). During this time, the minimum energy required to produce the radio ejection was $2.6\left(d \mathrm{kpc}^{-1}\right)^{8 / 7} \times 10^{42} \mathrm{erg}$, where the distance to XTE J1748-288, $d$, is less than $8 \mathrm{kpc}$. With an volume of $9 \times 10^{48} \mathrm{~cm}^{3}$, inferred from the outburst timescale, this implies an equipartition magnetic field on the order of $3 \mathrm{mG}$, similar to those in GRS $1915+105$ and SS 433.

These three objects are shown in Figure 4 by the cyan region labeled "XB". The combination of relatively low RM and mG fields again places $\nu_{\mathrm{SA}} \simeq 1 \mathrm{MHz}$. Taking a typical scale of $10^{16} \mathrm{~cm}$, this implies $\nu_{\mathrm{R}} \simeq 20 D_{16}^{1 / 3} \ell_{B, 16}^{-2 / 3} \mathrm{RM}_{2}^{1 / 3} \mathrm{kHz}$, and therefore refraction is insignificant in $\mathrm{XRB}$ jets.

\subsection{Galactic Center}

The inner $100 \mathrm{pc}$ of the Milky Way is an extraordinarily active region, exhibiting intense star formation (e.g., the Arches \& Quintuplet clusters), numerous supernovae remnants and the mysterious non-thermal filaments (NTF's). Unique to the Galactic center, the NTF's are comparatively bright, highly polarized filamentary synchrotron sources extending in some cases for nearly $30 \mathrm{pc}$. In addition to the large NTF's (the Southern \& Northern Threads, the Radio Arc and the Snake), there are a number of shorter NTF's, extending for 5, pc-10 pc, and in some cases misaligned with their larger brethren (Yusef- 
Zadeh \& Morris 1987; Lang et al. 1999; Law et al. 2008).

The origin of the NTF's is presently unclear. The orientation of the large NTF's appears to be aligned with a poloidal Galactic field, suggesting that they are associated with magnetic flux tubes that carry the Galactic field through the central regions. This is supported by the large observed polarizations, up to $70 \%$ in some places, which require the presence largescale ordered magnetic fields (see, e.g., Yusef-Zadeh et al. 1997). However, this is in conflict with the extraordinary magnetic fields required to maintain stability within the Galactic center's dynamic environment, nearly a $\mathrm{mG}$ (see, e.g., YusefZadeh \& Morris 1987). Furthermore, the presence of smaller, misaligned filaments appears to argue against a connection to large-scale Galactic fields (LaRosa et al. 2004).

The RM for the NTF's varies considerably along the filament, ranging from roughly $10^{2} \mathrm{rad} \mathrm{m}^{-2}$ to $6 \times 10^{3} \mathrm{rad} \mathrm{m}^{-2}$ (Yusef-Zadeh \& Morris 1987; Morris \& Yusef-Zadeh 1989; Gray et al. 1995; Yusef-Zadeh et al. 1997; Lang et al. 1999). These are quite large in comparison to the RM's of roughly $200 \mathrm{rad} \mathrm{m}^{-2}$ (implying field strengths of roughly $20 \mu \mathrm{G}$ ) observed for extragalactic sources that are viewed through the inner $170 \mathrm{pc}$ of the Galactic center (Roy et al. 2008), suggesting that the Faraday screen must lie close to, or even are intrinsic to, the NTF's. This is supported by the large RM gradients observed across some NTF's, which require turbulent $10 \mathrm{mG}$ ISM fields in the region, field strengths nearly an order of magnitude larger than those inferred in the NTF's themselves (YusefZadeh et al. 1997).

As a result, similar to Pulsars and AGN, there is considerable uncertainty regarding the region responsible for the observed Faraday rotation, and therefore the relevant magnetic field strength. Furthermore, given the dynamic nature of the Galactic center region it is additionally unclear if the field is ordered or turbulent. The red region labeled "GC" in the middle of Figure 4 shows the best guess values for the NTF's the Northern Thread, the Snake, the Radio Arc and G 359.54+0.18, and roughly characterizes the uncertainty in the magnetic field. By comparison, taking a fiducial NTF transverse scale of $0.1 \mathrm{pc}$ and line-of-sight length of $3 \mathrm{pc}$ gives a typical $\nu_{\mathrm{R}} \simeq 40 \mathrm{kHz}$, well below $\nu_{\mathrm{SA}}$. This is only weakly dependent upon the location of the Galactic center Faraday screen, rising to $0.1 \mathrm{MHz}$ for distances of up to $100 \mathrm{pc}$.

\subsection{Galactic Molecular Clouds}

Magnetic turbulence is thought to be critical for supporting structures within molecular clouds and generating the dense molecular cores that are the sites of star formation (McKee $\&$ Ostriker 2007). Despite this, there is still considerable uncertainty regarding the magnetic field strengths and geometry within individual objects, and therefore the role that magnetic fields play in the process of star formation. Typical values of the magnetic field in Molecular clouds range from Galactic value of a few $\mu \mathrm{G}$ to nearly a $\mathrm{mG}$ inferred from Zeeman splitting of molecular lines (see, e.g. Crutcher 1999; Han \& Zhang 2007). The measured molecular lines are presumably produced in the densest regions, and therefore indicative of the fields nearest the sites of star formation. Nevertheless, the comparative lack of large magnetic fields in stars implies that at some point during the star formation process the magnetic fields must somehow dissipate (either by diffusion or reconnection), and thus these may not represent the strongest magnetic fields present in molecular clouds.

High-resolution maps of the rotation measures of molecular clouds have been obtained via $21 \mathrm{~cm}$ observations of nearby star forming regions (see, e.g., Uyaniker et al. 2003; Wolleben $\&$ Reich 2004). These generally find considerable substructure and exhibit large RM gradients. The average average rotation measures are on the order of $30 \mathrm{rad} \mathrm{m}^{2}$, though with a dispersion of roughly $3 \times 10^{2} \mathrm{rad} \mathrm{m}^{2}$ about the mean value, and reaching $10^{3} \mathrm{rad} \mathrm{m}^{-2}$ in some places. This puts molecular clouds in the lower-left of Figure 4, denoted by the dark red region and labeled "MC", and $\nu_{\mathrm{SA}} \simeq 30 \mathrm{kHz}-2 \mathrm{MHz}$, reflecting the uncertainty in the underlying physical conditions.

As with other sources, the value of $\nu_{\mathrm{SA}}$ would all but determine the location of the Faraday rotating medium. Furthermore, comparing $\mathrm{RM}_{\mathrm{SA}}$ and $\mathrm{RM}_{\mathrm{S}}$ would provide an independent measurement of the magnetohydrodynamic turbulence believed to support these clouds. In particular, this would allow a comparison between the geometry of the magnetic field and that expected from the turbulent velocity field.

As with other sources, uncertainty arises in $\nu_{\mathrm{R}}$ associated with the uncertain scales of the location of and variations in the Faraday screen. Nevertheless, even for large molecular clouds $\left(10^{2} \mathrm{pc}\right)$ and relatively small turbulent structures $(0.1 \mathrm{pc})$ the refractive decoherence frequency is still only roughly $60 \mathrm{kHz}$, almost certainly well below the relevant estimates for $\nu_{\mathrm{SA}}$.

\subsection{Starburst Galaxies}

Infrared galaxies are presumed to be undergoing a phase of prodigious star formation. Therefore, one might expect that these offer the prospect of measuring the properties of extragalactic star forming regions generally, and extragalactic molecular clouds specifically. Radio observations of infrared galaxies have indeed shown typical RM's of $10 \mathrm{rad} \mathrm{m}^{-2}$, though with a dispersion of roughly $50 \mathrm{radm}^{-2}$ (though some reach nearly $200 \mathrm{rad} \mathrm{m}^{-2}$ ), these are somewhat more narrowly distribute about the mean (Heald et al. 2009). This is a reflection of the fact that we are necessarily averaging over a much larger region than we can resolve in Galactic molecular clouds. Indeed, the RM for the individual galaxies is comparable to that averaged over individual Galactic molecular clouds (Uyaniker et al. 2003; Wolleben \& Reich 2004).

Again there is considerable uncertainty in the strength of the magnetic field in the star forming regions of infrared galaxies. We adopt a typical value of $10 \mu \mathrm{G}$, though the dynamics of recent galactic interactions may raise this substantially. Nevertheless, the region occupied in Figure 4 is between molecular clouds and the ISM, labeled "IG" and shown in magenta. This puts $\nu_{\mathrm{SA}} \simeq 10^{2} \mathrm{kHz}$. This is, however, becoming uncomfortably close to the refractive decoherence scale, comparable to the $60 \mathrm{kHz}$ obtained in section 5.5. Thus, in this case even detection of the super-adiabatic regime will shed light upon the conditions within the star factories of starburst galaxies.

\subsection{Intracluster Medium}

The magnetic field embedded in the hot, turbulent gas at the centers of galaxy clusters has an estimated strength of $10 \mu \mathrm{G}-$ $30 \mu \mathrm{G}$. Combined with the kpc path lengths, dense cluster cores are capable of produce substantial RM's.

Indeed, the fact that $\mathrm{H} z \mathrm{RG}$ 's are located at the centers of cooling-core clusters suggests that the anomalously large RM's observed in these sources may be associated with the intracluster medium (ICM) and are not intrinsic to the galactic nuclei. This produces estimates for the magnetic field 1-2 orders of magnitude lower than those obtained in Section 4.3, roughly on the order of $20 \mu \mathrm{G}$. These are shown in the dark- 
orange region labeled "HzRG-c", implying that in the restframe $\nu_{\mathrm{SA}} \simeq 1 \mathrm{MHz}$ for these galaxies. Considering the high redshifts of these sources, this gives $\nu_{\mathrm{SA}} \simeq 300 \mathrm{kHz}$. This is well above typical estimates of $\nu_{\mathrm{R}}$, which for a $20 \mathrm{kpc}$ core exhibiting large-scale structure on $100 \mathrm{pc}$ scales is still roughly only $20 \mathrm{kHz}$.

Observations of typical radio galaxies embedded in the centers of clusters find rotation measures as high as $3 \times$ $10^{3} \mathrm{radm}^{-2}$ (Ge \& Owen 1993, 1994). Representative of these is $\mathrm{J} 1346+268$, located near the center of Abell 1795. Combined with electron density estimates from X-ray observations, this gives a lower-limit upon the cluster magnetic field strength of $20 \mu \mathrm{G}$. This is roughly an order of magnitude lower than the ICM equipartition value, suggesting that the magnetic field is likely to be tangled on scales small in comparison to the cluster size. Producing the observed RM with a stochastic field requires roughly $N=10^{2}$, resulting in an order of magnitude difference in $\mathrm{RM}_{\mathrm{SA}}$ and $\mathrm{RM}_{\mathrm{S}}$. However, the corresponding $\nu_{\mathrm{SA}}$ is roughly $300 \mathrm{kHz}$, comparable to that found for the $\mathrm{Hz} \mathrm{RG}$, assuming that the Faraday rotation occurs within the ICM in those sources.

\section{CONCLUSIONS}

Despite commonly being described in terms of the independent propagation of plasma modes, non-adiabatic effects at magnetic field reversals force the mode crossings that are critical to understanding the standard expressions for Faraday rotation. For nearly all astrophysical systems, at frequencies above a few $\mathrm{GHz}$, these effects dominate the propagation at magnetic field reversals. However, at sufficiently low frequencies it is possible to enter a "super-adiabatic" regime, in which the two plasma modes propagate independently throughout, resulting in rotation measures that are proportional to $\int n_{e}\left|B_{\|}\right| d \ell$ instead of $\int n_{e} B_{\|} d \ell$.

For large-scale ordered magnetic fields, there is little difference between the standard and super-adiabatic regimes. However, for magnetic field geometries exhibiting many reversals along the line of sight, the rotation measure in the superadiabatic regime can be substantially larger than that determined at high frequencies. Thus, comparisons between the standard and super-adiabatic rotation provides a unique means to measure the magnetic field geometry along the line of sight.

The frequency at which Faraday rotation transitions between the standard and super-adiabatic regimes, $\nu_{\mathrm{SA}}$, is itself dependent upon the local plasma properties at the magnetic field reversals, though only weakly dependent upon the magnetic field geometry. Thus even the detection of super-adiabatic Faraday rotation, marked by a rapid increase in the rotation measure at low frequencies, provides localized information about the plasma density and magnetic fields. This has the potential to remove the degeneracy between propagation path length and plasma density or magnetic field strength, allowing an unambiguous determination of the location of the Faraday screen in many systems.

For known systems, $\nu_{\mathrm{SA}}$ ranges from the heliospheric cutoff, approximately $10 \mathrm{kHz}$, to nearly $10 \mathrm{GHz}$, with regions containing stronger magnetic fields and higher rotation measures transitioning at higher frequencies. For resolved active galactic nuclei, such as Sgr A*, M81* and M31, this lies above the ionospheric cutoff at $15 \mathrm{GHz}$, and hence is observable from ground based radio telescopes. Some unresolved AGN, HzRG and Pulsars may be observable from the ground as well. Detection of super-adiabatic Faraday rotation in any of these sources would provide striking confirmation of the presence of in situ Faraday rotation. For X-ray binaries, infrared galaxies, molecular clouds, the Galactic center region, intracluster medium, interstellar medium and the solar corona, the super-adiabatic regime should be observable from space-based radio observatories.

The search for super-adiabatic Faraday rotation is especially timely given the proliferation of ultra-low frequency telescopes, such as the Murchison Widefield Array in Australia $(80 \mathrm{GHz}-300 \mathrm{GHz})$ and the Low Frequency Array in Europe $(10 \mathrm{MHz}-240 \mathrm{MHz})$, built ostensibly for the purpose of mapping $21 \mathrm{~cm}$ emission from the high- $z$ Universe, and the Frequency Agile Solar Radiotelescope in North America $(50 \mathrm{MHz}-21 \mathrm{GHz})$, currently under development (Bastian 2003). All of these devices will collect the full compliment of Stokes parameters, and thus will be capable of searching for super-adiabatic Faraday rotation. In the far term, space-based radio observatories, such as Astronomical Low Frequency Array, have been discussed for more than two decades now (Jones et al. 2000). They provide the only means to access the $10 \mathrm{kHz}-$ $15 \mathrm{MHz}$ radio window, and beyond the confines of the Earth, can in principle do so with resolutions comparable to much shorter wavelengths at the Very Long Baseline Array. The ability to directly probe the geometry of astrophysical magnetic fields along the line of sight should add to the list of science motivators for such a capability.

\section{ACKNOWLEDGEMENTS}

This research has made use of data from the MOJAVE database that is maintained by the MOJAVE team (Lister et al., 2009 , AJ, 137, 3718). This work was supported in part by the US Department of Energy under contract number DE-AC02$76 \mathrm{SF} 00515$.

\section{APPENDIX}

\section{RADIATIVE TRANSFER REGIMES}

In general, Maxwell's equations give

$$
\left(\nabla^{2}-\nabla \nabla+\omega^{2} \boldsymbol{\epsilon}\right) \cdot \mathbf{E}=0
$$

for an electric field $\mathbf{E}$, and a dielectric tensor $\epsilon$. For plane waves propagating along the $z$-axis in a plane parallel medium (we address the possibility of refraction in the following appendix), this reduces to

$$
\frac{d^{2} \mathbf{F}}{d z^{2}}+\omega^{2} \boldsymbol{\epsilon} \cdot \mathbf{F}=0
$$

where $\mathbf{F}$ is the Jones vector (i.e., a two-dimensional vector constructed from the transverse components of $\mathbf{E}$ ). For an anisotropic dielectric tensor, there will exist two nondegenerate transverse modes defined such that

$$
\omega^{2} \epsilon \mathbf{F}_{i}=k_{i}^{2} \mathbf{F}_{i} .
$$

In the case of a plasma, these are in general elliptically polarized and aligned with the component of the background magnetic field normal to the direction of propagation, i.e.,

$$
\mathbf{F}_{1}=\mathbf{Q}\left(\begin{array}{c}
\sin \chi \\
i \cos \chi
\end{array}\right) \text { and } \quad \mathbf{F}_{2}=\mathbf{Q}\left(\begin{array}{c}
\cos \chi \\
-i \sin \chi
\end{array}\right) \text {, }
$$

where the orientation of the polarization ellipses with respect to a set of axis fixed in space is determined by

$$
\mathbf{Q}=\left(\begin{array}{cc}
\cos \phi & \sin \phi \\
-\sin \phi & \cos \phi
\end{array}\right) \text {. }
$$


Then, $\mathbf{F}=F_{1} \mathbf{F}_{1}+F_{2} \mathbf{F}_{2}$, may be inserted into Equation (A2) to give

$$
\begin{gathered}
F_{1}^{\prime \prime}+2 i s_{2} \varphi F_{1}^{\prime}+\left(k_{1}^{2}-\varphi^{2}-\psi^{2}+i s_{2} \varphi^{\prime}+2 i c_{2} \varphi \psi\right) F_{1} \\
=2\left(\psi-i c_{2} \varphi\right) F_{2}^{\prime}+\left(\psi^{\prime}-i c_{2} \varphi^{\prime}+2 i s_{2} \varphi \psi\right) F_{2} \\
F_{2}^{\prime \prime}-2 i s_{2} \varphi F_{2}^{\prime}+\left(k_{2}^{2}-\varphi^{2}-\psi^{2}-i s_{2} \varphi^{\prime}-2 i c_{2} \varphi \psi\right) F_{2} \\
=-2\left(\psi+i c_{2} \varphi\right) F_{1}^{\prime}-\left(\psi^{\prime}+i c_{2} \varphi^{\prime}-2 i s_{2} \varphi \psi\right) F_{1},
\end{gathered}
$$

where a prime denotes differentiation with respect to $z, c_{2} \equiv$ $\cos 2 \chi, s_{2} \equiv \sin 2 \chi, \varphi \equiv \phi^{\prime}$ and $\psi \equiv \chi^{\prime}$. When $\varphi=0$, these reproduce Försterling's coupled equations (cf. Budden 1961; Ginzburg 1970).

Thus far, no approximations have been made regarding the wave length or scale lengths of the plasma. From the form of Equations (A6), it is clear that if $\phi$ and $\psi$ vanish, the two modes will propagate completely independently. In the limit that $\varphi$ and $\psi$ are small in comparison to $k_{1,2}$, we may look for WKB solutions of the form

$$
F_{i}=\frac{f_{i}}{\sqrt{k_{i}}} \mathrm{e}^{i \int k_{i} d z}
$$

and hence

$$
\begin{aligned}
& f_{1}^{\prime}+i s_{2} \varphi f_{1}=\left(\psi-i c_{2} \varphi\right) f_{2} \mathrm{e}^{-i \int \Delta k d z} \\
& f_{2}^{\prime}-i s_{2} \varphi f_{2}=-\left(\psi+i c_{2} \varphi\right) f_{1} \mathrm{e}^{i \int \Delta k d z}
\end{aligned}
$$

where $\Delta k \equiv k_{1}-k_{2}$ terms on the order of $\psi^{2}, \varphi^{2}, \psi^{\prime}, \varphi^{\prime}, \psi \varphi$, and $f_{i}^{\prime \prime}$ were ignored as they are small by assumption relative to those that remain. Further expand the $f_{i}$ as

$$
f_{1}=u_{1} \mathrm{e}^{-i \int(\Delta k / 2) d z} \text { and } f_{2}=u_{2} \mathrm{e}^{i \int(\Delta k / 2) d z} .
$$

Then,

$$
\begin{aligned}
& u_{1}^{\prime}-i\left(\frac{\Delta k}{2}-s_{2} \varphi\right) u_{1}=\left(\psi-i c_{2} \varphi\right) u_{2} \\
& u_{2}^{\prime}+i\left(\frac{\Delta k}{2}-s_{2} \varphi\right) u_{2}=-\left(\psi+i c_{2} \varphi\right) u_{1}
\end{aligned}
$$

which may be combined to give

$$
\begin{aligned}
& u_{1}^{\prime \prime}+\left[\left(\frac{\Delta k}{2}\right)^{2}+\psi^{2}+\varphi^{2}-s_{2} \varphi \Delta k-i \frac{\Delta k^{\prime}}{2}\right] u_{1}=0 \\
& u_{2}^{\prime \prime}+\left[\left(\frac{\Delta k}{2}\right)^{2}+\psi^{2}+\varphi^{2}-s_{2} \varphi \Delta k+i \frac{\Delta k^{\prime}}{2}\right] u_{2}=0 .
\end{aligned}
$$

If the $\psi$ and $\varphi$ terms are dominated by the $\Delta k$ terms, then

$$
u_{1,2} \simeq \text { const } \times \mathrm{e}^{ \pm i \int(\Delta k / 2) d z},
$$

and thus the $f_{i}$ are constant. Therefore, in this limit the modes propagate independently (the so-called adiabatic regime). In the opposing limit, when $\psi$ and $\varphi$ dominate $\Delta k$, then Equations (A11) are indistinguishable from the isotropic case (i.e., $\Delta k=$ 0 ), and therefore the polarization propagates unaltered (the socalled vacuum regime). This can be directly proved by solving for $u_{1,2}$ in this limit and expressing the answer in terms of $\mathbf{F}$. Because the $\varphi \Delta k$ term will only be relevant when

$$
\left(\frac{\Delta k}{2}\right) \varphi \sim \psi^{2}+\varphi^{2},
$$

this term may be neglected in determining the limiting regimes, yielding

$$
\begin{array}{ll}
\psi^{2}+\varphi^{2} \ll\left(\frac{\Delta k}{2}\right)^{2}+\left|\frac{\Delta k^{\prime}}{2}\right| & \text { adiabatic } \\
\psi^{2}+\varphi^{2} \gg\left(\frac{\Delta k}{2}\right)^{2}+\left|\frac{\Delta k^{\prime}}{2}\right| & \text { vacuum. }
\end{array}
$$

What remains is to identify these limits in terms of the physical characteristics of the plasma. That $\nu$ exceeds the upper cutoff of the extraordinary mode requires $X+Y<1$, which in turn requires that the propagation occur above both the electron cyclotron and electron plasma frequencies. Under these circumstances the ionic contribution to the dielectric tensor may be safely ignored. Thus, the $k_{1,2}$ are given by the standard Appleton-Hartree dispersion relations:

$$
k_{1,2}=\omega \sqrt{1-\frac{2 X(1-X)}{2(1-X)-Y^{2} \sin ^{2} \theta \mp \Gamma}}
$$

where

$$
\Gamma=\sqrt{Y^{4} \sin ^{4} \theta+4 Y^{2}(1-X)^{2} \cos ^{2} \theta} .
$$

To second order in $Y$, this gives

$$
\Delta k \simeq \frac{\omega}{c} X Y \sqrt{\cos ^{2} \theta+\left(\frac{Y}{2}\right)^{2} \sin ^{2} \theta} .
$$

The ellipticity angle is given by (see e.g. Ginzburg 1970; Budden 1964)

$$
\tan \chi=x+\operatorname{sgn}(x) \sqrt{1+x^{2}}
$$

where

$$
x \equiv \frac{Y \sin ^{2} \theta}{2(1-X) \cos \theta}
$$

As a direct result,

$$
\chi^{\prime}=\frac{x^{\prime}}{2\left(1+x^{2}\right)} \simeq \frac{Y}{4} \frac{\left(1+\cos ^{2} \theta\right) \sin \theta}{(Y / 2)^{2}+\cos ^{2} \theta} \theta^{\prime},
$$

where the finally expression assumes that $Y \ll 1$ and $X^{\prime}, Y^{\prime} \ll$ $\theta^{\prime}$. Note that for $\theta \simeq \pi / 2, \chi^{\prime} \simeq Y^{-1} \theta^{\prime} \simeq Y^{-1} \phi^{\prime}$ which at high frequencies $(Y \ll 1)$ is generally much larger than and $\phi^{\prime}$, and thus $\psi$ dominates $\varphi$ near magnetic field reversals in the lefthand sides of eqs. (A14), and in particular is maximized at $\theta=\pi / 2$.

On the other hand, as long as $X^{\prime}, Y^{\prime} \ll \phi$ near $\theta=\pi / 2$, $\left|\Delta k^{\prime} / 2\right|$ vanishes identically, due to the symmetry of $\Delta k$. This does not mean that $\left|\Delta k^{\prime} / 2\right|$ doesn't dominate $(\Delta k / 2)^{2}$ elsewhere, which is clearly the case for the parameters shown in Figure 1 . Nevertheless, sufficiently close to $\theta=\pi / 2$, the righthand sides of eqs. (A14) are dominated by the $(\Delta k / 2)^{2}$ term, and reach their minimum.

Therefore, for the mode propagation to remain adiabatic for all magnetic field orientations (i.e., through an entire magnetic field reversal), it is both necessary and sufficient to have

$$
\psi \ll\left|\frac{\Delta k}{2}\right| .
$$

It is this condition that we use to define the "super-adiabatic" regime. Note that at magnetic reversals

$$
\left|\frac{\Delta k}{2}\right| \phi \ll\left|\frac{\Delta k}{2}\right| \psi<\max \left[\psi^{2},\left(\frac{\Delta k}{2}\right)^{2}\right],
$$

and therefore, much smaller than the dominant term in the above adiabatic condition, either $\psi^{2}$ or $(\Delta k / 2)^{2}$, as was previously claimed. 


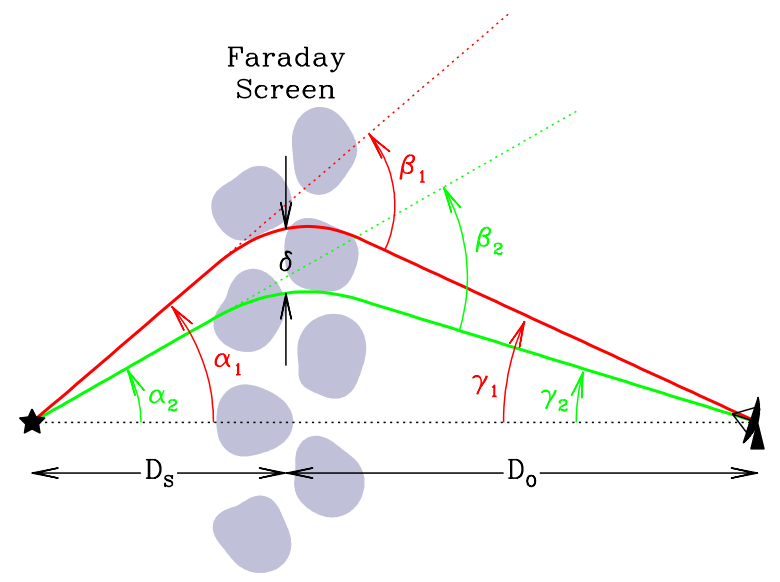

FIG. 5.- Diagram of the relevant angles and distances within the thinscreen approximation for the fast (green) and slow (red) modes. The maximum deviation is $\delta$, located within the screen itself.

\section{REFRACTION AND FARADAY DECOHERENCE}

In the previous section we ignored the refraction of the radio wave. For propagation near the plasma and/or cyclotron frequencies this is not justified. Therefore, here we address the corrections and limits that refraction places upon the computation of the Faraday rotation.

Refraction potentially enters into the problem in two ways. First it provides a third scale over which the plasma eigenmodes change, though typically larger than $2 \pi / \chi^{\prime}$ and $2 \pi / \phi^{\prime}$, and thus not relevant. Second, and much more important, is the possibility that the two plasma eigenmodes refract differently, propagating through significantly different regions and sampling distinct plasma conditions. It is this latter effect that we concern ourselves.

Let us define $\mathbf{x}_{1,2}(\eta)$ and $\mathbf{k}_{1,2}(\eta)$ be the trajectories and wavevectors of the two plasma eigenmodes, parametrized such that at $\eta=0$ they begin at the source and at $\eta=1$ they arrive at the observer. The phase difference between the two modes is then

$$
\int_{0}^{1} d \eta\left(\mathbf{k}_{1} \cdot \frac{d \mathbf{x}_{1}}{d \eta}-\mathbf{k}_{2} \cdot \frac{d \mathbf{x}_{2}}{d \eta}\right)
$$

That this may be reduced to an integral along a single curve, e.g., $\mathbf{x}=\left(\mathbf{x}_{1}+\mathbf{x}_{2}\right) / 2$, requires that the two curves never separate further than the local plasma correlation length, roughly $\ell_{B}$, leading to refractive decoherence. Note that in this case, it is not the total diffraction of the trajectories, but the differential trajectory that matters. Generally, this is a complicated global condition, requiring the complete construction of $\mathbf{x}_{1,2}$ prior to addressing. However, in the "thin-screen" approximation, we can obtain a simple condition for when refractive decoherence may be safely ignored.

Within the thin-screen approximation we assume that the Faraday screen's width is much smaller than both, the distance between the source and the screen, $D_{S}$, and the distance between the screen and the observer, $D_{O}$. Within this approximation, the trajectories are roughly straight lines, joined by a lensing angle at the screen. Geometrically, it is easy to see that $D_{S} \alpha_{i}=D_{O} \gamma_{i}$ and $\alpha_{i}+\gamma_{i}=\beta_{i}$, from which it follows directly that,

$$
\delta=\frac{D_{S} D_{O}}{D_{S}+D_{O}}\left(\beta_{1}-\beta_{2}\right) .
$$

That the maximum deviation between the paths taken by the two plasma eigenmodes is less than the characteristic plasma correlation length reduces to $\delta \ll \ell_{B}$, or

$$
\left|\beta_{1}-\beta_{2}\right| \ll \frac{D_{S}+D_{O}}{D_{S} D_{O}} \ell_{B}=\frac{\ell_{B}}{D},
$$

where $D^{-1} \equiv D_{S}^{-1}+D_{O}^{-1}$. At this point, we must determine $\beta_{i}$.

It suffices to consider propagation in the quasi-longitudinal regime since this dominates the differential refraction (because the difference in the indices of refraction is largest and the propagation nearly always occurs in this regime). Therefore, the dispersion relation is given by

$$
D_{1,2} \simeq \frac{1}{2}\left[k_{1,2}^{2}-\omega^{2}+\omega^{2} X(1 \pm Y)\right],
$$

i.e., $D_{1,2}$ vanishes along the ray. The equations defining the ray are

$$
\begin{gathered}
\frac{d \mathbf{x}_{1,2}}{d \eta}=\frac{\partial D_{1,2}}{\partial \mathbf{k}_{1,2}}=\mathbf{k}_{1,2}, \\
\frac{d \mathbf{k}_{1,2}}{d \eta}=-\frac{\partial D_{1,2}}{\partial \mathbf{x}_{1,2}}=-\frac{\omega^{2}}{2} \frac{\partial X(1 \pm Y)}{\partial \mathbf{x}_{1,2}} .
\end{gathered}
$$

Outside of the Faraday screen, $X=Y=0$, and $k_{i}^{2}=\omega^{2}$. In the weak deflection limit, we may treat the component of $\mathbf{k}_{i}$ along the original direction as unchanged, the refraction simply introducing an orthogonal component. That is, we set

$$
\beta_{1,2} \simeq-\omega^{-1} \int d \eta \frac{d \mathbf{x}_{1,2}}{d \eta} \times \frac{\partial X(1 \pm Y)}{\partial \mathbf{x}_{1,2}} \simeq-\frac{1}{2} X(1 \pm Y) f
$$

where $f$ is the characteristic fractional variation in the plasma parameters on the scale of $\ell_{B}$. Therefore, for a Faraday screen of width $\ell_{B}$ we obtain $\left|\beta_{1}-\beta_{2}\right| \simeq X Y f$. Should the screen be composed of may turbulent cells, the trajectories will diffuse, with $\left|\beta_{1}-\beta_{2}\right| \simeq \sqrt{N} X Y f \simeq \sqrt{L / \ell_{B}} X Y f$. Therefore, for the maximum deviation between the paths of the two plasma eigenmodes to be sufficiently small we require

$$
X Y f \ll \frac{\ell_{B}^{3 / 2}}{D L^{1 / 2}} .
$$

As with the super-adiabatic condition, we may view this physically, as a lower limit upon $\ell_{B}$, or observationally, as an upper limit upon $\nu$. In terms of the latter, we have

$$
\nu \gg\left(\nu_{P}^{2} \nu_{B} f \frac{D L^{1 / 2}}{\ell_{B}^{3 / 2}}\right)^{1 / 3}
$$

the expression underlying those in Equation (13).

\section{REFERENCES}

Agol, E. 2000, ApJ, 538, L121

Arons, J. \& Barnard, J. J. 1986, ApJ, 302, 120

Bahcall, J. N. 1989, Neutrino astrophysics (Cambridge University Press, Cambridge)

Barnard, J. J. \& Arons, J. 1986, ApJ, 302, 138

Bastian, T. S. 2003, Advances in Space Research, 32, 2705

Blandford, R. D. \& Konigl, A. 1979, ApJ, 232, 34

Bougeret, J.-L. 1996, Advances in Space Research, 18, 35 
Brocksopp, C., Miller-Jones, J. C. A., Fender, R. P., \& Stappers, B. W. 2007, MNRAS, 378, 1111

Broderick, A. E. \& Loeb, A. 2009, ApJ, 703, L104

Broderick, J. W., De Breuck, C., Hunstead, R. W., \& Seymour, N. 2007, MNRAS, 375, 1059

Brunthaler, A., Bower, G. C., \& Falcke, H. 2006, A\&A, 451, 845

Budden, K. G. 1961, Radio Waves in the Ionosphere (Cambridge University Press, Cambridge)

Budden, K. G. 1964, Lectures on Magnetoionic Theory (Gordon and Breach, New York)

Crutcher, R. M. 1999, ApJ, 520, 706

Doeleman, S. S. et al. 2008, Nature, 455,78

Falcke, H. \& Markoff, S. 2000, A\&A, 362, 113

Faraday, M. 1846, Phil. Trans. Roy. Soc., 136, 1

Frey, S., Gurvits, L. I., Paragi, Z., et al. 2008, A\&A, 477, 781

Ge, J. \& Owen, F. N. 1994, AJ, 108, 1523

Ge, J. P. \& Owen, F. N. 1993, AJ, 105, 778

Ghez, A. M. et al. 2008, ApJ, 689, 1044

Gillessen, S. et al. 2009, ApJ, 692, 1075

Gilmore, W. \& Seaquist, E. R. 1980, AJ, 85, 1486

Ginzburg, V. L. 1970, The propagation of electromagnetic waves in plasmas (Pergamon, Oxford)

Gray, A. D., Nicholls, J., Ekers, R. D., \& Cram, L. E. 1995, ApJ, 448, 164

Han, J. L. \& Zhang, J. S. 2007, A\&A, 464, 609

Heald, G., Braun, R., \& Edmonds, R. 2009, A\&A, 503, 409

Jones, D. L. et al. 2000, Advances in Space Research, 26, 743

Jorstad, S. G. et al. 2005, AJ, 130, 1418

Kharb, P., Gabuzda, D. C., O'Dea, C. P., Shastri, P., \& Baum, S. A. 2009, ApJ, 694, 1485

Lang, C. C., Morris, M., \& Echevarria, L. 1999, ApJ, 526, 727

LaRosa, T. N., Nord, M. E., Lazio, T. J. W., \& Kassim, N. E. 2004, ApJ, 607, 302

Law, C. J., Yusef-Zadeh, F., \& Cotton, W. D. 2008, ApJS, 177, 515

Li, Z., Wang, Q. D., \& Wakker, B. P. 2009, MNRAS, 397, 148

Lister, M. L. 2001, ApJ, 562, 208

Lister, M. L., Marscher, A. P., \& Gear, W. K. 1998, ApJ, 504, 702

Lister, M. L. \& Smith, P. S. 2000, ApJ, 541, 66

Lister, M. L. et al. 2009, AJ, 137, 3718
Loeb, A. \& Waxman, E. 2007, Journal of Cosmology and Astro-Particle Physics, 3, 11

Macquart, J.-P., Bower, G. C., Wright, M. C. H., Backer, D. C., \& Falcke, H. 2006, ApJ, 646, L111

Manchester, R. N., Hobbs, G. B., Teoh, A., \& Hobbs, M. 2005, AJ, 129, 1993, http://www.atnf.csiro.au/research/pulsar/psrcat

Mancuso, S. \& Spangler, S. R. 2000, ApJ, 539, 480

Marrone, D. P., Moran, J. M., Zhao, J.-H., \& Rao, R. 2007, ApJ, 654, L57

McKee, C. F. \& Ostriker, E. C. 2007, ARA\&A, 45, 565

Men, H., Ferrière, K., \& Han, J. L. 2008, A\&A, 486, 819

Miller-Jones, J. C. A., McCormick, D. G., Fender, R. P., et al. 2005, MNRAS, 363,867

Morris, M. \& Yusef-Zadeh, F. 1989, ApJ, 343, 703

Noutsos, A., Johnston, S., Kramer, M., \& Karastergiou, A. 2008, MNRAS, 386,1881

Perley, R. A. \& Taylor, G. B. 1991, AJ, 101, 1623

Porcas, R. W. \& Rioja, M. J. 2002, in Proceedings of the 6th EVN Symposium, ed. E. Ros, R. W. Porcas, A. P. Lobanov, \& J. A. Zensus, 65

Quataert, E. \& Gruzinov, A. 2000, ApJ, 545, 842

Roy, S., Pramesh Rao, A., \& Subrahmanyan, R. 2008, A\&A, 478, 435

Stirling, A. M., Spencer, R. E., Cawthorne, T. V., \& Paragi, Z. 2004, MNRAS, 354,1239

Taylor, G. B. 1998, ApJ, 506, 637

Taylor, G. B. 2000, ApJ, 533, 95

Taylor, G. B. \& Perley, R. A. 1992, A\&A, 262, 417

Thompson, C., Blandford, R. D., Evans, C. R., \& Phinney, E. S. 1994, ApJ, 422,304

Udomprasert, P. S., Taylor, G. B., Pearson, T. J., \& Roberts, D. H. 1997, ApJ, 483, L9

Uyaniker, B., Landecker, T. L., Gray, A. D., \& Kothes, R. 2003, ApJ, 585, 785

Wolleben, M. \& Reich, W. 2004, A\&A, 427, 537

Yuan, F., Quataert, E., \& Narayan, R. 2003, ApJ, 598, 301

Yusef-Zadeh, F. \& Morris, M. 1987, ApJ, 322, 721

Yusef-Zadeh, F., Wardle, M., \& Parastaran, P. 1997, ApJ, 475, L119

Zavala, R. T. \& Taylor, G. B. 2003, ApJ, 589, 126

Zavala, R. T. \& Taylor, G. B. 2004, ApJ, 612, 749 